\title{
Calderón-Zygmund operators with operator-valued kernel on homogeneous Besov spaces
}

\author{
Cornelia Kaiser*1 \\ ${ }^{1}$ Mathematisches Institut I, Universität Karlsruhe, Englerstraße 2, 76128 Karlsruhe, Germany
}

\begin{abstract}
Key words vector-valued Besov spaces, Calderón-Zygmund operators, T(1) theorem MSC (2000) Primary: 42B20, 46E40; Secondary: 46E35

We consider generalized Calderón-Zygmund operators whose kernel takes values in the space of all continuous linear operators between two Banach spaces. In the spirit of the $T(1)$ theorem of David and Journé we prove boundedness results for such operators on vector-valued Besov spaces.
\end{abstract}

\section{Introduction}

Singular integral operators between spaces of vector-valued functions (i.e., functions that take their values in some Banach space $X$ ) have been studied extensively in recent years. A major break-through were theorems dealing with operator-valued Fourier multipliers between Lebesgue-Bochner spaces (e.g. $[18,8])$. These theorems require a special geometry of the underlying Banach spaces (the UMD property) and a stronger notion of uniform boundedness, the so-called R-boundedness. In particular, they are not applicable for non-reflexive Banach spaces. However it was observed by H. Amann [1] and L. Weis [17] that these restrictions do not apply when working with vector-valued Besov spaces. In fact, Mihlin and Hörmander type Fourier multiplier theorems were obtained for the Besov spaces $B_{p}^{s, q}(X)$, where $X$ is an arbitrary Banach space $[6,8]$. Taking the point of view of convolution operators instead of Fourier multipliers, T. Hytönen and L. Weis [11] have proved boundedness results for translation-invariant operators on $B_{p}^{s, q}(X)$. Again one does not need to impose any conditions on the geometry of $X$.

In this paper we want to move away from translation invariant operators to the study of nonconvolution type singular integrals of the from

$$
(T f)(u)=\int_{\mathbb{R}^{N}} K(u, v) f(v) d v .
$$

Inspired by the famous $T(1)$ theorem of G. David and J.-L. Journé $[2,3]$ on the $L_{2}$ boundedness of operators (1.1) T. Figiel [4] has proved a $T(1)$ theorem for $X$-valued $L_{p}$ functions, where $X$ has the UMD-property. The kernels $K$ are still scalar valued, however.

Recently T. Hytönen and L. Weis [10] gave a new proof of Figiel's T(1) theorem and extended it to operator-valued kernels $K$. In addition to the geometric property of $X$ they have to impose Rboundedness conditions on the kernels that in general are stronger than just uniform boundedness. It is the main goal of this work to show how the main ideas in the proof of T. Hytönen and L. Weis can be used to obtain a $T(1)$ theorem on vector-valued homogeneous Besov spaces, where the kernel $K$ is operator-valued. Since we don't impose further conditions neither on the geometry of the Banach space nor on the boundedness of the kernels, our result is in the same line as the work on operator-valued Fourier multipliers and singular convolution operators described above. For an application of our result to wavelets on vector-valued Besov spaces see [13].

In the scalar-valued setting, various authors (e.g. $[5,7,14,15,19])$ obtained results of the same spirit as the $T(1)$ theorem of David and Journé for other scalar-valued function spaces, including homogeneous

* Corresponding author: e-mail: cornelia.kaiser@math.uni-karlsruhe.de, Phone: +49721608 8892, Fax: +497216087650 
Besov and Triebel-Lizorkin spaces. The present paper gives a new proof of the known scalar-valued results for homogeneous Besov spaces, which directly extends to the vector-valued situation. (In [9] and [12], we show that we can apply the same methods of proof to obtain $T(1)$ theorems in spaces of Triebel-Lizorkin type.) We note that, while Lemarie [14] and Torres [15] apply different methods, the ideas used by Han and Hofmann in [7] are similar to ours. Han and Hofmann only deal with Besov spaces with smoothness index $s$ in $(-1,1)$, however.

\section{Definitions and Notations}

Throughout this paper $X, Y$, and $Z$ are complex Banach spaces. The space $\mathcal{L}(X, Y)$ of bounded linear operators from $X$ to $Y$ is endowed with the uniform operator topology. $X^{\prime}=\mathcal{L}(X, \mathbb{C})$ denotes the dual space of $X$. All our (possibly vector-valued) functions and distributions will be defined on $\mathbb{R}^{N}$ for a fixed positive integer $N$. Therefore the various function spaces $E\left(\mathbb{R}^{N}, X\right)$ in this paper are denoted simply by $E(X)$. For example we write $L_{p}(X)$ for the Bochner-Lebesgue space $L_{p}\left(\mathbb{R}^{N}, X\right), p \in[1, \infty]$, equipped with its usual norm.

$\mathbb{N}:=\{0,1,2, \ldots\}$ is the set of all nonnegative integers. The conjugate exponent $p^{\prime}$ of $p \in[1, \infty]$ is given by $\frac{1}{p}+\frac{1}{p^{\prime}}=1$. For a multiindex $\alpha \in \mathbb{N}^{N}$, we write $|\alpha|=\alpha_{1}+\cdots+\alpha_{N}$. Also $u^{\alpha}=u_{1}^{\alpha_{1}} \cdots u_{N}^{\alpha_{N}}$ for $u=\left(u_{1}, \ldots, u_{N}\right) \in \mathbb{R}^{N}$ and $\partial_{u}^{\alpha}=\partial_{u_{N}}^{\alpha_{N}} \cdots \partial_{u_{1}}^{\alpha_{1}}$ where $\partial_{u_{i}}=\frac{\partial}{\partial u_{i}}$.

We write $\mathcal{D}\left(\mathbb{R}^{N}, X\right)$ for the space of all compactly supported smooth functions with values in $X$. The Schwartz class $\mathcal{S}\left(\mathbb{R}^{N}, X\right)$ is the space of all $X$-valued rapidly decreasing smooth functions, endowed with its usual topology. For $\mathcal{D}\left(\mathbb{R}^{N}, \mathbb{C}\right)$ and $\mathcal{S}\left(\mathbb{R}^{N}, \mathbb{C}\right)$ we also write $\mathcal{D}\left(\mathbb{R}^{N}\right)$ and $\mathcal{S}\left(\mathbb{R}^{N}\right)$ respectively. The space $\mathcal{S}^{\prime}\left(\mathbb{R}^{N}, X\right)$ of all $X$-valued tempered distributions is defined as the space of all continuous linear operators from $\mathcal{S}\left(\mathbb{R}^{N}\right)$ to $X$.

The Fourier transform $\mathcal{F}: \mathcal{S}\left(\mathbb{R}^{N}\right) \rightarrow \mathcal{S}\left(\mathbb{R}^{N}\right)$ is defined by

$$
(\mathcal{F} \varphi)(u)=\widehat{\varphi}(u)=\int_{\mathbb{R}^{N}} e^{-i u v} \varphi(v) d v .
$$

\section{The operator $T$}

We start from a continuous linear operator

$$
T: \mathcal{S}\left(\mathbb{R}^{N}\right) \rightarrow \mathcal{S}^{\prime}\left(\mathbb{R}^{N}, \mathcal{L}(X, Y)\right) .
$$

The operator $T$ can be identified with the continuous bilinear form

$$
\mathcal{S}\left(\mathbb{R}^{N}\right) \times \mathcal{S}\left(\mathbb{R}^{N}\right) \rightarrow \mathcal{L}(X, Y), \quad(\varphi, \psi) \mapsto(T \varphi)(\psi)
$$

In place of $(T \varphi) \psi$ we also use the more suggestive notation $\langle\psi, T \varphi\rangle$. To $T$ we assign an "adjoint" operator

$$
T^{\prime}: \mathcal{S}\left(\mathbb{R}^{N}\right) \rightarrow \mathcal{S}^{\prime}\left(\mathbb{R}^{N}, \mathcal{L}\left(Y^{\prime}, X^{\prime}\right)\right), \quad\left\langle\psi, T^{\prime} \varphi\right\rangle:=\langle\varphi, T \psi\rangle^{\prime},
$$

where the latter' designates the usual Banach adjoint of an operator in $\mathcal{L}(X, Y)$.

\section{The associated kernel}

Suppose now that $K:\left\{(u, v) \in \mathbb{R}^{N} \times \mathbb{R}^{N}: u \neq v\right\} \rightarrow \mathcal{L}(X, Y)$ is continuous. We say that $T$ is a singular integral operator associated with $K$ if

$$
\langle\varphi, T \phi\rangle=\int_{\mathbb{R}^{N}} \varphi(u) \int_{\mathbb{R}^{N}} K(u, v) \phi(v) d v d u
$$

holds for all $\varphi, \phi \in \mathcal{D}\left(\mathbb{R}^{N}\right)$ with $\operatorname{supp} \varphi \cap \operatorname{supp} \phi=\emptyset$. This means that, for each $\phi \in \mathcal{D}\left(\mathbb{R}^{N}\right)$, the distribution $T \phi$ agrees almost everywhere on the complement of $\operatorname{supp} \phi$ with the continuous function $\int_{\mathbb{R}^{N}} K(\cdot, v) \phi(v) d v$, defined on the complement of $\operatorname{supp} \phi$. It is clear from $(2.1)$ that $T^{\prime}$ is a singular integral operator with associated kernel $K^{\prime}$ given by $K^{\prime}(u, v)=K(v, u)^{\prime}$ for $u \neq v$. 
The class $\mathrm{CZO}_{n+\nu}$

For $n \in \mathbb{N}$ and $\nu \in(0,1]$ we consider the standard estimates

$\left(\mathrm{SE}_{n}\right) K$ is continuously differentiable up to order $n$ with respect to the first variable and

$$
\mathcal{C}_{\alpha}(K)=\sup \left\{|u-v|^{N+|\alpha|}\left\|\left(\partial_{u}^{\alpha} K\right)(u, v)\right\|: u \neq v\right\}
$$

is finite for all multiindices $\alpha$ with $|\alpha| \leq n$;

$\left(\mathrm{SE}_{n+\nu}\right) K$ is continuously differentiable up to order $n$ with respect to the first variable and

$$
\mathcal{C}_{\alpha, \nu}(K)=\sup \left\{|u-v|^{N+n+\nu} \frac{\left\|\left(\partial_{u}^{\alpha} K\right)(u, v)-\left(\partial_{u}^{\alpha} K\right)\left(u_{0}, v\right)\right\|}{\left|u-u_{0}\right|^{\nu}}:\right\}
$$

is finite for some multiindex $\alpha$ with $|\alpha|=n$.

We say that $T \in \mathrm{CZO}_{n+\nu}$ if $T$ is a singular integral operator with associated kernel $K$ satisfying $\left(\mathrm{SE}_{n}\right)$ and $\left(\mathrm{SE}_{n+\nu}\right)$. Note that $T \in \mathrm{CZO}_{n+\nu}$ does not emply that $T^{\prime} \in \mathrm{CZO}_{n+\nu}$.

If $0<\nu<\delta<1$, then

$$
\left(\mathrm{SE}_{n+1}\right) \Longrightarrow\left(\mathrm{SE}_{n+\delta}\right) \Longrightarrow\left(\mathrm{SE}_{n+\nu}\right) .
$$

Therefore $\mathrm{CZO}_{n+\nu}$ is contained in $\mathrm{CZO}_{m+\mu}$ if $n+\nu \geq m+\mu$.

We will also use a weaker version of $\left(\mathrm{SE}_{n+\nu}\right)$, namely the following Hörmander type condition for $t \in[1, \infty)$ :

$\left(\mathrm{HE}_{n+\nu, t}\right) K$ is continuously differentiable up to order $n$ with respect to the first variable and

$$
\mathcal{C}_{\alpha, \nu, t}(K)=\sup \left\{\begin{array}{c}
\frac{\rho^{|\alpha|+\nu+N / t^{\prime}}}{\left|u-u_{0}\right|^{\nu}}\left(\int_{\rho<|u-v|<2 \rho}\left\|\left(\partial_{u}^{\alpha} K\right)(u, v)-\left(\partial_{u}^{\alpha} K\right)\left(u_{0}, v\right)\right\|^{t} d v\right)^{1 / t}: \\
\rho>2\left|u-u_{0}\right|
\end{array}\right\}
$$

is finite for some multiindex $\alpha$ with $|\alpha|=n$.

Observe that, if $1 \leq s<t<\infty$, then

$$
\left(\mathrm{SE}_{n+\nu}\right) \Longrightarrow\left(\mathrm{HE}_{n+\nu, t}\right) \Longrightarrow\left(\mathrm{HE}_{n+\nu, s}\right) \text {. }
$$

We write

$$
\mathcal{C}_{n+\nu}(K):=\min _{|\alpha|=n} \mathcal{C}_{\alpha, \nu}(K), \quad \mathcal{C}_{n+\nu, t}(K):=\min _{|\alpha|=n} \mathcal{C}_{\alpha, \nu, t}(K) .
$$

\section{Definition of $T\left(u^{\alpha}\right)$}

The action of $T \in \mathrm{CZO}_{n+\nu}$ is not a priori defined on the function $u \mapsto u^{\alpha} \notin \mathcal{S}\left(\mathbb{R}^{N}\right)$, but we can make sense of the notion $T\left(u^{\alpha}\right)$ for $|\alpha| \leq n$ : We will define $T\left(u^{\alpha}\right)$ as a linear operator acting on

$$
\mathcal{D}^{n}\left(\mathbb{R}^{N}\right):=\left\{\varphi \in \mathcal{D}\left(\mathbb{R}^{N}\right): \int_{\mathbb{R}^{N}} u^{\alpha} \varphi(u) d u=0 \text { for all }|\alpha| \leq n\right\} .
$$

For doing this we first observe that, if $\varphi \in \mathcal{D}^{n}\left(\mathbb{R}^{N}\right)$, the distribution $(\cdot)^{\alpha} T^{\prime} \varphi$ agrees with an integrable function on the complement of any neighborhood of $\operatorname{supp} \varphi$. A proof of this fact can be found in Section 4 (Lemma 4.2). Now choose $\psi \in \mathcal{D}\left(\mathbb{R}^{N}\right)$ such that $\psi \equiv 1$ in a fixed neighborhood of $\operatorname{supp} \varphi$ and define

$$
\left\langle(\cdot)^{\alpha}, T^{\prime} \varphi\right\rangle:=\left\langle(\cdot)^{\alpha} \psi, T^{\prime} \varphi\right\rangle+\int_{\mathbb{R}^{N}}(1-\psi(u)) u^{\alpha}\left(T^{\prime} \varphi\right)(u) d u .
$$

Here the first term is given by the usual pairing between test functions and distributions and the second term exists because $(\cdot)^{\alpha} T^{\prime} \varphi$ is integrable on the support of $1-\psi$. One can show that the value of $\left\langle(\cdot)^{\alpha}, T^{\prime} \varphi\right\rangle$ is independent of the actual choice of $\psi$. Now we make the natural definition $\left\langle\varphi, T\left(u^{\alpha}\right)\right\rangle:=\left.\left\langle(\cdot)^{\alpha}, T^{\prime} \varphi\right\rangle^{\prime}\right|_{X} \in \mathcal{L}(X, Y)$. 


\section{The weak boundedness property}

The closed ball with center $u \in \mathbb{R}^{N}$ and radius $r>0$ is denoted by $\bar{B}(u, r)$. We say that $\varphi$ is a normalized bump function associated with the unit ball $\bar{B}(0,1)$ if $\varphi \in \mathcal{D}\left(\mathbb{R}^{N}\right)$ with $\operatorname{supp} \varphi \subseteq \bar{B}(0,1)$ and $\left\|D^{\alpha} \varphi\right\|_{L_{\infty}} \leq 1$ for all $|\alpha| \leq M$, where $M$ is a large fixed number. ${ }^{1} \phi$ is a normalized bump function associated with the ball $\bar{B}(u, r)$ if $\phi(\cdot)=r^{-N} \varphi\left(r^{-1}(\cdot-u)\right)$, where $\varphi$ is a normalized bump function associated with the unit ball.

The operator $T$ is said to have the weak boundedness property provided that, for every pair of normalized bump functions $\varphi, \phi$ associated with any ball $\bar{B}(u, r)$ we have $\|\langle\phi, T \varphi\rangle\| \leq C r^{-N}$.

The weak boundedness property describes the behavior of $\|\langle\phi, T \varphi\rangle\|$ if the two bump functions are associated with the same ball. But can one also say something if the two bump functions are associated with balls of different size and location? The following result gives an answer to this question. Since the proof is rather long and technical we postpone it to the last section of this paper. (For $n=0$, part (b) of Lemma 2.1 was proved in [10, Sect. 2].)

Lemma 2.1 Let $k \in \mathbb{N}, a>0, w \in \mathbb{R}^{N}$, and let $\varphi, \phi \in \mathcal{D}^{n}\left(\mathbb{R}^{N}\right)$ be normalized bump functions associated with $\bar{B}(0, a)$ and $\bar{B}\left(w, 2^{k} a\right)$ respectively.

(a) If $T \in \mathrm{CZO}_{\nu}$ satisfies the weak boundedness property, then there is a constant $C_{1}<\infty$ such that for all $v \in \mathbb{R}^{N}$

$$
\|\langle\phi(\cdot-v), T[\varphi(\cdot-v)]\rangle\| \leq C_{1} \frac{1+k}{\left(a 2^{k}\right)^{N}}\left(1+\frac{|w|}{a 2^{k}}\right)^{-N-\nu} .
$$

(b) If $T \in \mathrm{CZO}_{n+\nu}$ satisfies the weak boundedness property and the condition $T\left(u^{\alpha}\right)=0$ for all $|\alpha| \leq n$, then there are constants $C_{2}<\infty$ and $\delta>0$ such that for all $v \in \mathbb{R}^{N}$,

$$
\|\langle\varphi(\cdot-v), T[\phi(\cdot-v)]\rangle\| \leq C_{2}\left(a 2^{k}\right)^{-N-n-\nu}\left(1+\frac{|w|}{a 2^{k}}\right)^{-N-\delta} .
$$

\section{A resolution of unity}

We will decompose our operator $T$ into parts we can handle using Lemma 2.1. For this decomposition we use test functions with special properties described in Lemma 2.2 below. For $k=0$, Lemma 2.2 was proved in [10, Sect. 2].

Lemma 2.2 Let $k \in \mathbb{N}$. Then there exist $\Phi \in \mathcal{D}^{k}\left(\mathbb{R}^{N}\right)$ and such that the following properties hold:

- $\Phi$ is radial and real-valued,

- $\widehat{\Phi}, \widehat{\Psi} \geq 0$,

- $\widehat{\Phi}(u) \geq 1$ for $\frac{1}{2} \leq|u| \leq 2$,

- $\widehat{\Psi}$ is supported in $\left\{\frac{1}{2} \leq|u| \leq 2\right\}$,

- $\sum_{j \in \mathbb{Z}} \widehat{\Phi}\left(2^{j} u\right) \widehat{\Psi}\left(2^{j} u\right)=1$ for all $u \in \mathbb{R}^{N} \backslash\{0\}$.

Proof. Take any non-zero, real, radial $\varphi \in \mathcal{D}^{k}\left(\mathbb{R}^{N}\right)$. (For the existence of such a $\varphi$ see Remark 2.3 below.) Since $\varphi$ is even, its Fourier transform is real. Then also $\phi:=\varphi * \varphi$ is real, radial, and in $\mathcal{D}^{k}\left(\mathbb{R}^{N}\right)$. Moreover, $\widehat{\phi}=\widehat{\varphi}^{2} \geq 0$. Since $\widehat{\phi}$ is radial and not identically vanishing, it is strictly positive on some annulus $0<a_{1} \leq|u| \leq a_{2}$. Taking as $\Phi$ a suitable linear combination, with positive coefficients, of dilates of $\varphi$, we ensure the condition $\widehat{\Phi} \geq 1$ for $\frac{1}{2} \leq|u| \leq 2$.

Let $\widehat{\psi}$ be non-negative, supported in $\left\{\frac{1}{2} \leq|u| \leq 2\right\}$, and with $\sum_{j \in \mathbb{Z}} \psi\left(2^{j} u\right)=1$ for $u \neq 0$. Now set $\widehat{\Psi}(u):=\widehat{\psi}(u) / \widehat{\Phi}(u)$ on $\left\{\frac{1}{2} \leq|u| \leq 2\right\}$ and 0 otherwise.

\footnotetext{
1 If we are dealing with operators in $\mathrm{CZO}_{n+\nu}$, we always choose $M>n$.
} 
With $\Phi, \Psi$ as in Lemma 2.2 and $j \in \mathbb{Z}$, we denote

$$
\Phi_{j}(u):=2^{-N j} \Phi\left(2^{-j} u\right), \quad \Psi_{j}(u):=2^{-N j} \Psi\left(2^{-j} u\right)
$$

and

$$
P_{j} f:=\Phi_{j} * f, \quad Q_{j} f=\Psi_{j} * f, \quad f \in \mathcal{S}^{\prime}\left(\mathbb{R}^{N}, X\right) .
$$

Remark 2.3 In the proof of Lemma 2.2 we used a non-zero, real, radial $\varphi \in \mathcal{D}^{k}\left(\mathbb{R}^{N}\right)$. To see that such $\varphi$ exists, let $\omega \in \mathcal{D}^{k+N-1}(\mathbb{R})$ be non-zero, real, and have its support in $(0, \infty)$. Then using polar coordinates one can show that $\int_{\mathbb{R}^{N}} u^{\alpha} \omega(|u|) d u=0$ for all $|\alpha| \leq k$. Now take any non-zero, real, radial $\phi \in \mathcal{D}\left(\mathbb{R}^{N}\right)$ and let $\varphi=\omega(|\cdot|) * \phi$. Then $\varphi$ has the desired properties.

\section{Calderón-Zygmund operators on Besov spaces}

Let $\mathcal{Z}\left(\mathbb{R}^{N}, X\right)$ be the space of all Schwartz functions $\varphi \in \mathcal{S}\left(\mathbb{R}^{N}, X\right)$ such that $\left(\partial^{\alpha} \widehat{\varphi}\right)(0)=0$ for all multiindices $\alpha \in \mathbb{N}^{N}$. Then $\mathcal{Z}\left(\mathbb{R}^{N}, X\right)$ is a closed subspace of $\mathcal{S}\left(\mathbb{R}^{N}, X\right)$. If $\mathcal{Z}^{\prime}\left(\mathbb{R}^{N}, X\right)$ denotes the space of all continuous linear operators from $\mathcal{Z}\left(\mathbb{R}^{N}\right)=\mathcal{Z}\left(\mathbb{R}^{N}, \mathbb{C}\right)$ to $X$, then $\mathcal{S}^{\prime}\left(\mathbb{R}^{N}, X\right) / \mathcal{P}\left(\mathbb{R}^{N}, X\right)$ and $\mathcal{Z}^{\prime}\left(\mathbb{R}^{N}, X\right)$ are isomorphic (cf. [16, 5.1.2] and [11, Section 7]). Here $\mathcal{P}\left(\mathbb{R}^{N}, X\right)$ stands for the space of all polynomials on $\mathbb{R}^{N}$ with coefficients in $X$.

\section{Homogeneous Besov spaces}

Let $\phi \in \mathcal{S}\left(\mathbb{R}^{N}\right)$ such that $\widehat{\phi}$ is radial, equal to 1 in $\bar{B}(0,1)$, and supported in $\bar{B}(0,2)$. Now set $\varphi=$ $\phi-\frac{1}{2^{N}} \widehat{\phi}\left(\frac{\dot{2}}{2}\right)$ and $\varphi_{j}=2^{-N j} \varphi\left(2^{-j} \cdot\right), j \in \mathbb{Z}$. Then $\sum_{j \in \mathbb{Z}} \widehat{\varphi_{j}}(\xi)=1$, if $\xi \neq 0$.

Let $p, q \in[1, \infty]$ and $s \in \mathbb{R}$. The homogeneous Besov spaces $\dot{B}_{p}^{s, q}(X)=\dot{B}_{p}^{s, q}\left(\mathbb{R}^{N}, X\right)$ is the space consisting of all $f \in \mathcal{Z}^{\prime}\left(\mathbb{R}^{N}, X\right)$ such that

$$
\|f\|_{\dot{B}_{p}^{s, q}(X)}:=\left\|\left(2^{-j s}\left\|f * \varphi_{j}\right\|_{L_{p}(X)}\right)_{j \in \mathbb{Z}}\right\|_{\ell_{q}(\mathbb{Z})}
$$

is finite. As in the scalar-valued case one can show that different choices of $\varphi$ lead to equivalent norms and that $\dot{B}_{p}^{s, q}(X)$, endowed with $\|\cdot\|_{\dot{B}_{p}^{s, q}(X)}$, is a Banach space. Moreover, as in the scalar-valued case on can prove that

$$
\mathcal{Z}\left(\mathbb{R}^{N}, X\right) \subseteq \dot{B}_{p}^{s, q}\left(\mathbb{R}^{N}, X\right) \subseteq \mathcal{Z}^{\prime}\left(\mathbb{R}^{N}, X\right) .
$$

\section{Extension of $T$}

Let $X, Y$ be arbitrary Banach spaces and $T: \mathcal{S}\left(\mathbb{R}^{N}\right) \rightarrow \mathcal{S}^{\prime}\left(\mathbb{R}^{N}, \mathcal{L}(X, Y)\right)$ as in Section 2 . let

From $T$ we derive a linear mapping $\widetilde{T}: \mathcal{S}\left(\mathbb{R}^{N}\right) \otimes X \rightarrow \mathcal{S}^{\prime}\left(\mathbb{R}^{N}, Y\right):$ for $x \in X$ and $\varphi, \psi \in \mathcal{S}\left(\mathbb{R}^{N}\right)$, we

$$
\langle\psi, \widetilde{T}[\varphi \otimes x]\rangle:=\langle\psi, T \varphi\rangle x \in Y .
$$

This makes sense, since $\langle\psi, T \varphi\rangle \in \mathcal{L}(X, Y)$. So $\widetilde{T}[\varphi \otimes x]$ is a $Y$-valued tempered distribution and $\widetilde{T}$ is well-defined on $\mathcal{S}\left(\mathbb{R}^{N}\right) \times X$. Now we extend $\widetilde{T}$ to $\mathcal{S}\left(\mathbb{R}^{N}\right) \otimes X$ by linearity. In the following we will not distinguish between $T$ and $\widetilde{T}$.

\section{Main result}

Now we state our main result on the boundedness of singular integral operators on homogeneous Besov spaces.

Theorem 3.1 Let $n \in \mathbb{N}$ and $\nu \in(0,1)$. Suppose $T \in \mathrm{CZO}_{n+\nu}$ satisfies the weak boundedness property and the condition $T\left(u^{\alpha}\right)=0$ for all $|\alpha| \leq n$. Then

(a) $T$ extends to a bounded linear operator from $\dot{B}_{p}^{s, q}(X)$ to $\dot{B}_{p}^{s, q}(Y)$ for each $s \in(0, n+\nu)$ and each $p, q \in[1, \infty]$.

(b) If in addition $T^{\prime} \in \mathrm{CZO}_{n+\nu}$ and $T^{\prime}\left(u^{\alpha}\right)=0$ for all $|\alpha| \leq n$, then $T$ extends to a bounded linear operator from $\dot{B}_{p}^{s, q}(X)$ to $\dot{B}_{p}^{s, q}(Y)$ for each $|s|<n+\nu$ and each $p, q \in[1, \infty]$. 
For the proof we will use the resolution of unity introduced in Section 2. For $f \in \mathcal{S}\left(\mathbb{R}^{N}\right)$ and $j, k \in \mathbb{Z}$,

$$
\begin{aligned}
\left(P_{j} T P_{k} f\right)(u) & =\left(\Phi_{j} * T\left[\Phi_{k} * f\right]\right)(u)=\left\langle\Phi_{j}(u-\cdot), T\left[\int_{\mathbb{R}} \Phi_{k}(\cdot-v) f(v) d v\right]\right\rangle \\
& =\int_{\mathbb{R}}\left\langle\Phi_{j}(u-\cdot), T\left[\Phi_{k}(\cdot-v)\right]\right\rangle f(v) d v=\int_{\mathbb{R}} K_{j, k}(u, v) f(v) d v
\end{aligned}
$$

where $K_{j, k}(u, v):=\left\langle\Phi_{j}(\cdot-u), T\left[\Phi_{k}(\cdot-v)\right]\right\rangle \in \mathcal{L}(X, Y)$. (Recall that $\Phi$ is radial.)

In a first step, we show that the operators $T_{j, k}$ associated with the kernels $K_{j, k}$ are bounded from $L_{p}(X)$ to $L_{p}(Y)$ for all $p \in[1, \infty]$.

Proposition 3.2 Suppose $T \in \mathrm{CZO}_{n+\nu}$ satisfies the weak boundedness condition and $T\left(u^{\alpha}\right)=0$ for $|\alpha| \leq n$. The operator $T_{j+k, j}, j, k \in \mathbb{Z}$, defined by

$$
T_{j+k, j} f=\int_{\mathbb{R}^{N}} K_{j+k, j}(u, v) f(v) d v, \quad f \in L_{p}(X) \cap L_{\infty}(X)
$$

extends to a bounded linear operator from $L_{p}(X)$ to $L_{p}(Y)$ for all $p \in[1, \infty]$. Moreover there is a constant $C$ such that, for all $j, k \in \mathbb{Z}$,

$$
\left\|T_{j+k, j}\right\| \leq \begin{cases}C(1+k), & k \geq 0 \\ C 2^{k(n+\nu)}, & k \leq 0\end{cases}
$$

If in addition $T^{\prime} \in \mathrm{CZO}_{n+\nu}$ and $T^{\prime}\left(u^{\alpha}\right)=0$ for $|\alpha| \leq n$, then we have the estimate

$$
\left\|T_{j+k, j}\right\| \leq C 2^{-|k|(n+\nu)} .
$$

Pr o of. We consider the case $k \geq 0$. Then, for each $u \in \mathbb{R}^{N}$,

$$
\begin{aligned}
\int_{\mathbb{R}^{N}}\left\|K_{j+k, j}(u, u-v)\right\| d v & =\int_{\mathbb{R}^{N}}\left\|\left\langle\Phi_{j+k}(\cdot-u), T\left[\Phi_{j}(\cdot-(u-v))\right]\right\rangle\right\| d v \\
& \leq \frac{C_{1}(1+k)}{\left(a 2^{j+k}\right)^{N}} \int_{\mathbb{R}^{N}}\left(1+\frac{|v|}{a 2^{j+k}}\right)^{-N-\nu} d v \\
& =C_{1}(1+k) \int_{\mathbb{R}^{N}}(1+|v|)^{-N-\nu} d v
\end{aligned}
$$

Here we have used Part (a) of Lemma 2.1 with $\varphi=\Phi_{j}$ associated to $\bar{B}\left(0,2^{j} a\right)$ and $\phi=\Phi_{j+k}(\cdot-v)$ associated to $\bar{B}\left(v, 2^{k} 2^{j} a\right)$, where $a$ is chosen such that $\operatorname{supp} \Phi \subseteq \bar{B}(0, a)$.

If $k \leq 0$, we use Part (b) of Lemma 2.1 with $\varphi=\Phi_{j+k}$ associated to $\bar{B}\left(0,2^{j+k} a\right)$ and $\phi=\Phi_{j}(\cdot-v)$ associated to $\bar{B}\left(0,2^{-k} 2^{j+k} a\right)$ to obtain

$$
\begin{aligned}
\int_{\mathbb{R}^{N}}\left\|K_{j+k, j}(u, u-v)\right\| d v & =\int_{\mathbb{R}^{N}}\left\|\left\langle\Phi_{j+k}(\cdot-u), T\left[\Phi_{j}(\cdot-u+v)\right]\right\rangle\right\| d v \\
& \leq \frac{C_{2} 2^{k(n+\nu)}}{\left(a 2^{j}\right)^{N}} \int_{\mathbb{R}^{N}}\left(1+\frac{|v|}{a 2^{j}}\right)^{-N-\delta} d v \\
& =C_{2} 2^{k(n+\nu)} \int_{\mathbb{R}^{N}}(1+|v|)^{-N-\delta} d v .
\end{aligned}
$$

Let $f \in L_{p}(X) \cap L_{\infty}(X)$ and $j, k \in \mathbb{Z}$. Then for each $u \in \mathbb{R}^{N}$

$$
\int_{\mathbb{R}^{N}}\left\|K_{j+k, j}(u, u-v) f(u-v)\right\|_{Y} d v \leq \int_{\mathbb{R}^{N}}\left\|K_{j+k, j}(u, u-v)\right\| d v\|f\|_{L_{\infty}(X)} .
$$

Therefore $K_{j+k, j}(u, u-\cdot) f(u-\cdot) \in L_{1}(Y)$ for each $u \in \mathbb{R}^{N}$ and, by substitution,

$$
\int_{\mathbb{R}^{N}} K_{j+k, j}(u, u-v) f(u-v) d v=\int_{\mathbb{R}^{N}} K_{j+k, j}(u, v) f(v) d v .
$$


Hence we can estimate

$$
\begin{aligned}
\left\|\int_{\mathbb{R}^{N}} K_{j+k, j}(\cdot, v) f(v) d v\right\|_{L_{p}(Y)} & =\left\|\int_{\mathbb{R}^{N}} K_{j+k, j}(\cdot, \cdot-v) f(\cdot-v) d v\right\|_{L_{p}(Y)} \\
& \leq \int_{\mathbb{R}^{N}}\left\|K_{j+k, j}(\cdot, \cdot-v)\right\|\|f(\cdot-v)\|_{L_{p}(X)} d v \\
& \leq \begin{cases}C(1+k)\|f\|_{L_{p}(X)}, & k \geq 0, \\
C 2^{k(n+\nu)}\|f\|_{L_{p}(X)}, & k \leq 0 .\end{cases}
\end{aligned}
$$

Since $L_{p}(X) \cap L_{\infty}(X)$ is dense in $L_{p}(X)$ for each $p \in[1, \infty]$, the first part of the proposition is proved.

Now suppose that in addition $T^{\prime} \in \mathrm{CZO}_{n+\nu}$ and $T^{\prime}\left(u^{\alpha}\right)=0$ for $|\alpha| \leq n$. We only have to consider the case $k \geq 0$. For each $u \in \mathbb{R}^{N}$, we use Part (b) of Lemma 2.1 for $T^{\prime}$ to show that

$$
\int_{\mathbb{R}^{N}}\left\|K_{j+k, j}(u, u-v)\right\| d v \leq C 2^{-k(n+\nu)} \int_{\mathbb{R}^{N}}(1+|v|)^{-N-\delta} d v
$$

Now we proceed as above.

In the next proposition we take a closer look at the "building blocks" from which we will construct our operator.

Proposition 3.3 For every $f \in \dot{B}_{p}^{s, q}(X)$ and every $k \in \mathbb{Z}$, the series

$$
\sum_{l \in \mathbb{Z}} Q_{l+k} T_{l+k, l} Q_{l} f
$$

converges in $\dot{B}_{p}^{s, q}(Y)$ if $q<\infty$ and always in $\mathcal{Z}^{\prime}\left(\mathbb{R}^{N}, Y\right)$ to an element in $\dot{B}_{p}^{s, q}(Y)$. Moreover, we have the estimate

$$
\left\|\sum_{l \in \mathbb{Z}} Q_{l+k} T_{l+k, l} Q_{l} f\right\|_{\dot{B}_{p}^{s, q}(Y)} \leq \begin{cases}C(1+k) 2^{-k s}\|f\|_{\dot{B}_{p}^{s, q}(X)}, & k \geq 0 \\ C 2^{k(n+\nu-s)}\|f\|_{\dot{B}_{p}^{s, q}(X)}, & k \leq 0 .\end{cases}
$$

If in addition $T^{\prime} \in \mathrm{CZO}_{n+\nu}$ and $T^{\prime}\left(u^{\alpha}\right)=0$ for $|\alpha| \leq n$, then

$$
\left\|\sum_{l \in \mathbb{Z}} Q_{l+k} T_{l+k, l} Q_{l} f\right\|_{\dot{B}_{p}^{s, q}(Y)} \leq C 2^{-|k|(n+\nu-|s|)}\|f\|_{\dot{B}_{p}^{s, q}(X)} .
$$

To prove Proposition 3.3 we will need the following lemma, which gives an estimate of the $\ell_{q}\left(L_{p}(X)\right)$ norm of $\left(2^{-k s} Q_{k} f\right)_{k \in \mathbb{Z}}$ in terms of the Besov norm of $f$ :

Lemma 3.4 For all $p, q \in[1, \infty]$ and all $s \in \mathbb{R}$ we have that

$$
\left\|\left(2^{-k s}\left\|Q_{k} f\right\|_{L_{p}(X)}\right)_{k \in \mathbb{Z}}\right\|_{\ell_{q}(\mathbb{Z})} \leq C\|f\|_{\dot{B}_{p}^{s, q}(X)}, \quad f \in \dot{B}_{p}^{s, q}(X) .
$$

Proof. Let $\varphi$ be as in the definition of the homogeneous Besov spaces at the beginning of this section. Taking into account the support properties of $\widehat{\varphi}_{j}$ and $\widehat{\Psi}_{k}$ we have that $\varphi_{j} * \Psi_{k}=0$ if $|j-k| \geq 2$. Hence

$$
\left\|Q_{k} f\right\|_{L_{p}(X)}=\left\|\sum_{j=k-1}^{k+1} \Psi_{k} * \varphi_{j} * f\right\| \leq \sum_{j=k-1}^{k+1}\left\|\Psi_{k} * \varphi_{j} * f\right\|_{L_{p}(X)} \leq\|\Psi\|_{1} \sum_{j=k-1}^{k+1}\left\|\varphi_{j} * f\right\|_{L_{p}(X)}
$$

and

$$
\begin{aligned}
\left\|\left(2^{-k s}\left\|Q_{k} f\right\|_{L_{p}(X)}\right)_{k \in \mathbb{Z}}\right\|_{\ell_{q}(\mathbb{Z})} & \leq\|\Psi\|_{1}\left\|\left(2^{-k s} \sum_{j=k-1}^{k+1}\left\|\varphi_{j} * f\right\|_{L_{p}(X)}\right)_{k \in \mathbb{Z}}\right\|_{\ell_{q}(\mathbb{Z})} \\
& \leq\|\Psi\|_{1}\left(2^{-s}+1+2^{s}\right)\|f\|_{\dot{B}_{p}^{s, q}(X)} .
\end{aligned}
$$

This proves the lemma. 
Proof of Proposition 3.3. Again let $\varphi$ be as in the definition of the homogeneous Besov spaces. Let $k \in \mathbb{Z}$ be fixed and $F$ be a finite subset of $\mathbb{Z}$. Then

$$
\varphi_{j+k} * \sum_{l \in F} Q_{l+k} T_{l+k, l} Q_{l} f=\sum_{l \in F} \varphi_{j+k} * \Psi_{l+k} *\left(T_{l+k, l} Q_{l} f\right),
$$

and $\varphi_{j+k} * \Psi_{l+k}=0$ if $|j-l|>1$. Write $F_{j}=F \cap\{j-1, j, j+1\}$. Therefore, for $q<\infty$,

$$
\begin{aligned}
\left\|\varphi_{j+k} * \sum_{l \in F} Q_{l+k} T_{l+k, l} Q_{l} f\right\|_{L_{p}(Y)} & \leq \sum_{l \in F_{j}}\left\|\varphi_{j+k} * \Phi_{l+k}\right\|_{L_{1}}\left\|T_{l+k, l} Q_{l} f\right\|_{L_{p}(Y)} \\
& \leq c \sup _{l \in F_{j}}\left\|T_{l+k, l}\right\| \sum_{l \in F_{j}}\left\|Q_{l} f\right\|_{L_{p}(X)} \\
& \leq 3 c \sup _{l \in \mathbb{Z}}\left\|T_{l+k, l}\right\|\left(\sum_{l \in F_{j}}\left\|Q_{l} f\right\|_{L_{p}(X)}^{q}\right)^{1 / q}
\end{aligned}
$$

and

$$
\begin{aligned}
\sum_{j \in \mathbb{Z}} 2^{-(j+k) s q} & \left\|\varphi_{j+k} * \sum_{l \in F} Q_{l+k} T_{l+k, l} Q_{l} f\right\|_{L_{p}(Y)}^{q} \\
& \leq\left(3 c \sup _{l \in \mathbb{Z}}\left\|T_{l+k, l}\right\|\right)^{q} \sum_{j \in \mathbb{Z}} 2^{-(j+k) s q} \sum_{l \in F_{j}}\left\|Q_{l} f\right\|_{L_{p}(X)}^{q} \\
& \leq\left(3 c \cdot 2^{-k s} \sup _{l \in \mathbb{Z}}\left\|T_{l+k, l}\right\|\right)^{q}\left(2^{-s q}+1+2^{s q}\right) \sum_{j \in F} 2^{-j s q}\left\|Q_{j} f\right\|_{L_{p}(X)}^{q}
\end{aligned}
$$

This implies that the series (3.1) converges unconditionally in $\dot{B}_{p}^{s, q}(Y)$. Indeed, for $\varepsilon>0$ choose a finite subset of $F_{0}$ of $\mathbb{Z}$ such that for all finite subsets $F$ of $\mathbb{Z} \backslash\left\{F_{0}\right\}$ we have that

$$
\sum_{j \in F} 2^{-j s q}\left\|Q_{j} f\right\|_{L_{p}(X)}^{q} \leq \varepsilon^{q}
$$

Then

$$
\begin{aligned}
\left\|\sum_{l \in F} Q_{l+k} T_{l+k, l} Q_{l} f\right\|_{\dot{B}_{p}^{s, q}(Y)} & =\left(\sum_{j \in \mathbb{Z}} 2^{-(j+k) s q}\left\|\varphi_{j+k} * \sum_{l \in F} Q_{l+k} T_{l+k, l} Q_{l} f\right\|_{L_{p}(Y)}^{q}\right)^{1 / q} \\
& \leq c 2^{-k s} \sup _{l \in \mathbb{Z}}\left\|T_{l+k, l}\right\| \varepsilon
\end{aligned}
$$

where $c$ is some constant not depending on $F$. So the series (3.1) is shrinking and therefore unconditionally convergent.

For convergence of $(3.1)$ in $\mathcal{Z}^{\prime}\left(\mathbb{R}^{N}, Y\right)$, let $\psi \in \mathcal{Z}\left(\mathbb{R}^{N}\right)$. Then

$$
\begin{aligned}
\sum_{l \in \mathbb{Z}}\left\|\left\langle Q_{l+k} T_{l+k, l} Q_{l} f, \psi\right\rangle\right\|=\sum_{l \in \mathbb{Z}}\left\|\left\langle T_{l+k, l} Q_{l} f, Q_{l+k} \psi\right\rangle\right\| \\
\leq \sum_{l \in \mathbb{Z}}\left\|T_{l+k, l} Q_{l} f\right\|_{L_{p}(Y)}\left\|Q_{l+k} \psi\right\|_{L_{p^{\prime}}} \leq \sup _{l \in \mathbb{Z}}\left\|T_{l+k, l}\right\| \sum_{l \in \mathbb{Z}}\left\|Q_{l} f\right\|_{L_{p}(X)}\left\|Q_{l+k} \psi\right\|_{L_{p^{\prime}}} \\
=2^{-s k} \sup _{l \in \mathbb{Z}}\left\|T_{l+k, l}\right\| \sum_{l \in \mathbb{Z}} 2^{-s l}\left\|Q_{l} f\right\|_{L_{p}(X)} 2^{s(k+l)}\left\|Q_{l+k} \psi\right\|_{L_{p^{\prime}}}\left\|\left(2^{s j}\left\|Q_{j} f\right\|_{L_{p^{\prime}}(X)}\right)_{j \in \mathbb{Z}}\right\|_{\ell_{q^{\prime}(\mathbb{Z})}}
\end{aligned}
$$

is finite by Lemma 3.4 , and since $\psi \in \mathcal{Z}\left(\mathbb{R}^{N}\right) \subseteq \dot{B}_{p^{\prime}}^{-s, q^{\prime}}\left(\mathbb{R}^{N}\right)$. So we have proved convergence of (3.1) in $\mathcal{Z}^{\prime}\left(\mathbb{R}^{N}, Y\right)$. 
Replacing $F$ by $\mathbb{Z}$ in (3.2) and (3.3) and using Lemma 3.4 we obtain $\sum_{l \in \mathbb{Z}} Q_{l+k} T_{l+k, l} Q_{l} f \in \dot{B}_{p}^{s, q}(Y)$

$$
\left\|\sum_{l \in \mathbb{Z}} Q_{l+k} T_{l+k, l} Q_{l} f\right\|_{\dot{B}_{p}^{s, q}(Y)} \leq c 2^{-k s} \sup _{l \in \mathbb{Z}}\left\|T_{l+k, l}\right\|\|f\|_{\dot{B}_{p}^{s, q}(X)}
$$

for all $s \in \mathbb{R}^{N}$ and all $q, p \in[1, \infty]$. By Proposition 3.2,

$$
2^{-k s} \sup _{l \in \mathbb{Z}}\left\|T_{l+k, l}\right\| \leq \begin{cases}C(1+k) 2^{-k s}, & k \geq 0 \\ C 2^{k(n+\nu-s)}, & k \leq 0\end{cases}
$$

in the general case. If in addition $T^{\prime} \in \mathrm{CZO}_{n+\nu}$ and $T^{\prime}\left(u^{\alpha}\right)=0$ for $|\alpha| \leq n$, then

$$
2^{-k s} \sup _{l \in \mathbb{Z}}\left\|T_{l+k, l}\right\| \leq C 2^{-k s} 2^{-|k|(n+\nu)} \leq C 2^{-|k|(n+\nu-|s|)} .
$$

This proves the claimed estimates.

Finally we prove our main theorem on the boundedness of $T$ on homogeneous Besov spaces:

Proof of Theorem 3.1. (a) If $s \in(0, n+\nu)$, then by Proposition 3.3

$$
\sum_{k \in \mathbb{Z}}\left\|\sum_{l \in \mathbb{Z}} Q_{l+k} T_{l+k, l} Q_{l} f\right\|_{\dot{B}_{p}^{s, q}(Y)} \leq C\|f\|_{\dot{B}_{p}^{s, q}(X)} .
$$

Therefore

$$
U f:=\sum_{k \in \mathbb{Z}} \sum_{l \in \mathbb{Z}} Q_{l+k} T_{l+k, l} Q_{l} f
$$

converges in $\dot{B}_{p}^{s, q}(Y)$ and defines a bounded linear operator $U: \dot{B}_{p}^{s, q}(X) \rightarrow \dot{B}_{p}^{s, q}(Y)$.

It is easy to see that $U$ is an extension of $\widetilde{T}$. Indeed, let $f \in \mathcal{S}\left(\mathbb{R}^{N}\right) \otimes X$. Then

$$
U f=\sum_{k \in \mathbb{Z}} \sum_{l \in \mathbb{Z}} Q_{l+k} P_{l+k} T P_{l} Q_{l} f=\sum_{l \in \mathbb{Z}} \sum_{k \in \mathbb{Z}} Q_{k} P_{k} T P_{l} Q_{l} f=\tilde{T} f .
$$

(b) In the case that also $T^{\prime} \in \mathrm{CZO}_{n+\nu}$ and $T^{\prime}\left(u^{\alpha}\right)=0$ for $|\alpha| \leq n$, (3.4) holds for all $|s|<n+\nu$. Now we proceed as above.

Remark 3.5 In part (a) of Theorem 3.1, instead of assuming $T \in \mathrm{CZO}_{n+\nu}$, it is sufficient to $\left(\mathrm{SE}_{0}\right)$, $\left(\mathrm{SE}_{\nu}\right)$, and $\left(\mathrm{HE}_{n+\nu, t}\right)$ for some $t \in[1, \infty)$ with $\frac{N}{t}<n+\nu$. Part (b) is still true if one replaces the assumption $T, T^{\prime} \in \mathrm{CZO}_{n+\nu}$ by $\left(\mathrm{HE}_{n+\nu, t}\right)$ for both $T$ and $T^{\prime}$. This follows directly from the estimates in Section 4 used for the proof of Lemma 2.1.

Remark 3.6 In case (b) in Theorem 3.1, the operator $U$ from (3.5) is the only extension of $\tilde{T}$ that is not only $\mathcal{L}\left(\dot{B}_{p}^{s, q}(X), \dot{B}_{p}^{s, q}(Y)\right)$, but also $\sigma\left(\dot{B}_{p}^{s, q}(X), \dot{B}_{p^{\prime}}^{-s, q^{\prime}}\left(X^{\prime}\right)\right)$-to- $\sigma\left(\dot{B}_{p}^{s, q}(Y), \dot{B}_{p^{\prime}}^{-s, q^{\prime}}\left(Y^{\prime}\right)\right)$ continuous.

$U$ is indeed $\sigma\left(\dot{B}_{p}^{s, q}(X), \dot{B}_{p^{\prime}}^{-s, q^{\prime}}\left(X^{\prime}\right)\right)$-to- $\sigma\left(\dot{B}_{p}^{s, q}(Y), \dot{B}_{p^{\prime}}^{-s, q^{\prime}}\left(Y^{\prime}\right)\right)$ continuous, since $U^{\prime}$ maps $\dot{B}_{p^{\prime}}^{-s, q^{\prime}}\left(Y^{\prime}\right)$ to $\dot{B}_{p^{\prime}}^{-s, q^{\prime}}\left(X^{\prime}\right)$. To see this, observe that $\left.\left(T_{l+k, l}\right)^{\prime}\right|_{L_{p^{\prime}}\left(Y^{\prime}\right)} \in \mathcal{L}\left(L_{p^{\prime}}\left(Y^{\prime}\right), L_{p^{\prime}}\left(X^{\prime}\right)\right)$ with norm $\leq\left\|T_{l+k, l}\right\|$ (cf. the proof of Proposition 3.2). Now it can be shown as in Proposition 3.3 that, for $g \in \dot{B}_{p^{\prime}}^{-s, q^{\prime}}\left(Y^{\prime}\right)$,

$$
U^{\prime} g=\sum_{k \in \mathbb{Z}} \sum_{l \in \mathbb{Z}} Q_{l}\left(T_{l+k, l}\right)^{\prime} Q_{l+k} g \in \dot{B}_{p^{\prime}}^{-s, q^{\prime}}\left(X^{\prime}\right)
$$

Uniqueness follows from the fact that, for each Banach space $Z$, the closure of $\mathcal{S}\left(\mathbb{R}^{N}, Z\right)$ in $\dot{B}_{p}^{s, q}(Z)$ is $\sigma\left(\dot{B}_{p}^{s, q}(Z), \dot{B}_{p^{\prime}}^{-s, q^{\prime}}\left(Z^{\prime}\right)\right)$-dense in $\dot{B}_{p}^{s, q}(Z)$. 


\section{Some Technical Lemmas}

In this section let $n \in \mathbb{N}, \nu \in(0,1)$, and $t \in[1, \infty)$. We use the notation $a \lesssim b$ if there is a constant $c$, only depending on $N, n, \nu$, and $t$, such that $a \leq c b$.

We first state and prove a lemma we will find useful below. It deals with estimates for $T^{\prime}$ if applied to some bump function.

Lemma 4.1 Let $T$ be a singular integral operator with associated kernel $K$ satisfying $\left(\mathrm{HE}_{n+\nu, t}\right)$. If $R \geq 2 \rho>0$ and $\varphi \in \mathcal{D}^{n}\left(\mathbb{R}^{N}\right)$ with $\operatorname{supp} \varphi \subseteq \bar{B}(0, \rho)$, then

$$
\left(\frac{1}{R^{N}} \int_{R<|v|<2 R}\left\|\left(T^{\prime} \varphi\right)(v)\right\|^{t} d v\right)^{1 / t} \lesssim \mathcal{C}_{n+\nu, t}(K)\|\varphi\|_{L_{\infty}}\left(\frac{\rho}{R}\right)^{N+n+\nu} .
$$

In particular,

$$
\left(\frac{1}{R^{N}} \int_{R<|v|}\left\|v^{\beta}\left(T^{\prime} \varphi\right)(v)\right\|^{t} d v\right)^{1 / t} \lesssim \mathcal{C}_{n+\nu, t}(K)\|\varphi\|_{L_{\infty}} R^{|\beta|}\left(\frac{\rho}{R}\right)^{N+n+\nu}
$$

for all multiindices $\beta$ with $|\beta| \leq n$.

Proof. By mathematical induction, one can show that

$$
\mathcal{D}^{n}\left(\mathbb{R}^{N}\right)=\left\{\varphi \in \mathcal{D}\left(\mathbb{R}^{N}\right): \int_{\mathbb{R}^{N}}\left(\mathcal{I}^{\alpha} \varphi\right)(u) d u=0 \text { for all }|\alpha| \leq n\right\},
$$

where $\mathcal{I}^{\alpha} \varphi:=\mathcal{I}_{N}^{\alpha_{N}} \cdots \mathcal{I}_{1}^{\alpha_{1}} \varphi$ with

$$
\left(\mathcal{I}_{j} \varphi\right)(u):=\int_{-\infty}^{u_{j}} \varphi(v) d v_{j}, \quad j \in\{1, \ldots, N\} .
$$

Let $\alpha \in \mathbb{N}^{N}$ be chosen such that $\left(\mathrm{HE}_{n+\nu, t}\right)$ holds. Then

$$
\begin{aligned}
\int_{\mathbb{R}^{N}} K(u, v) \varphi(u) d u & =(-1)^{n} \int_{\mathbb{R}^{N}}\left(\partial_{u}^{\alpha} K\right)(u, v)\left(\mathcal{I}^{\alpha} \varphi\right)(u) d u \\
& =(-1)^{n} \int_{\bar{B}(0, \rho)}\left[\left(\partial_{u}^{\alpha} K\right)(u, v)-\left(\partial_{u}^{\alpha} K\right)(0, v)\right]\left(\mathcal{I}^{\alpha} \varphi\right)(u) d u
\end{aligned}
$$

Since

$$
\left(T^{\prime} \varphi\right)(v)=\int_{\mathbb{R}^{N}} K^{\prime}(v, u) \varphi(u) d u=\left[\int_{\mathbb{R}^{N}} K(u, v) \varphi(u) d u\right]^{\prime}
$$

for almost all $v \notin \bar{B}(0, \rho)$, we can estimate

$$
\begin{aligned}
& \left(\frac{1}{R^{N}} \int_{R<|v|<2 R}\left\|\left(T^{\prime} \varphi\right)(v)\right\|^{t} d v\right)^{1 / t} \\
& \leq \int_{B(0, \rho)}\left(\frac{1}{R^{N}} \int_{R<|u|<2 R}\left\|\left(\partial_{u}^{\alpha} K\right)(u, v)-\left(\partial_{u}^{\alpha} K\right)(0, v)\right\|^{t} d v\right)^{1 / t}\left|\left(\mathcal{I}^{\alpha} \varphi\right)(u)\right| d u \\
& \leq \mathcal{C}_{n+\nu, t}(K) \int_{B(0, \rho)} \frac{|u|^{\nu}}{R^{N+n+\nu}}\left|\left(\mathcal{I}^{\alpha} \varphi\right)(u)\right| d u \\
& \leq \mathcal{C}_{n+\nu, t}(K)\left\|\mathcal{I}^{\alpha} \varphi\right\|_{L_{1}} \frac{\rho^{\nu}}{R^{N+n+\nu}} .
\end{aligned}
$$

But $\left\|\mathcal{I}^{\alpha} \varphi\right\|_{L_{1}} \lesssim \rho^{N+n}\|\varphi\|_{L_{\infty}}$ and the first inequality is proved. 
Now let $\beta$ be a multiindex with $|\beta| \leq n$. Then

$$
\begin{aligned}
\left(\frac{1}{R^{N}}\right. & \left.\int_{R<|u|}\left\|v^{\beta}\left(T^{\prime} \varphi\right)(v)\right\|^{t} d v\right)^{1 / t} \\
& =\left(\sum_{k=0}^{\infty} \frac{1}{R^{N}} \int_{2^{k} R<|u|<2^{k+1} R}\left\|v^{\beta}\left(T^{\prime} \varphi\right)(v)\right\|^{t} d v\right)^{1 / t} \\
& \lesssim \mathcal{C}_{n+\nu, t}(K)\|\varphi\|_{L_{\infty}}(2 R)^{|\beta|}\left(\frac{\rho}{R}\right)^{N+n+\nu}\left(\sum_{k=0}^{\infty} 2^{k t\left(|\beta|-n-\nu-N / t^{\prime}\right)}\right)^{1 / t}
\end{aligned}
$$

Since $\sum_{k=0}^{\infty} 2^{k t\left(|\beta|-n-\nu-N / t^{\prime}\right)} \leq \sum_{k=0}^{\infty} 2^{-k t \nu}$, the second assertion is proved, too.

The following corollary we use in our definition of $T\left(u^{\alpha}\right)$ :

Corollary 4.2 Let $T$ be a singular integral operator with associated kernel $K$ satisfying condition $\left(\mathrm{HE}_{n+\nu, 1}\right)$. Let $\varphi \in \mathcal{D}^{n}\left(\mathbb{R}^{N}\right), V$ some neighborhood of $\operatorname{supp} \varphi$, and $\beta$ a multiindex with $|\beta| \leq n$. Then, on the complement of $V,(\cdot)^{\beta} T^{\prime} \varphi$ agrees with an integrable function.

Pr o of. Choose $\rho>0$ such that $\operatorname{supp} \varphi \subseteq \bar{B}(0, \rho)$. Now Lemma 4.1 implies that

$$
\int_{2 \rho<|v|}\left\|v^{\beta}\left(T^{\prime} \varphi\right)(v)\right\| d v<\infty
$$

Since $K$ is continuous and $\bar{B}(0,2 \rho) \cap V^{c}$ is compact and disjoint from $\operatorname{supp} \varphi$,

$$
\int_{\bar{B}(0,2 \rho) \cap V^{c}}\left\|v^{\beta}\left(T^{\prime} \varphi\right)(v)\right\| d v<\infty
$$

So the corollary is proved.

We assume that all bump functions used in this section satisfy the appropriate estimates on the derivatives up to order $M$, where $M>n$ (cf. Section 2).

The operator $T$ is said to have the weak boundedness property at 0 provided that, for every pair of normalized bump functions $\varphi, \phi$ associated with any ball $\bar{B}(0, r)$ we have $\|\langle\phi, T \varphi\rangle\| \leq C r^{-N}$. The infimum over all such constants $C$ is denoted by $\mathcal{C}_{w . b . p .}(T)$.

Lemma 4.3 Let $k \in \mathbb{N}, w \in \mathbb{R}^{N}$, and let $\varphi, \phi \in \mathcal{D}^{0}\left(\mathbb{R}^{N}\right)$ be normalized bump functions associated with $\bar{B}(0,1)$ and $\bar{B}\left(w, 2^{k}\right)$ respectively. If $T \in \mathrm{CZO}_{\nu}$ satisfies the weak boundedness property at 0 , then

$$
\|\langle\phi, T \varphi\rangle\| \lesssim \mathcal{C}_{1}(T) \frac{1+k}{2^{k N}}\left(1+\frac{|w|}{2^{k}}\right)^{-N-\nu}
$$

Here $\mathcal{C}_{1}(T)=\max \left\{\mathcal{C}_{w . b . p .}(T), \mathcal{C}_{0}(K), \mathcal{C}_{\nu}(K)\right\}$

Pr o of. Let $\psi \in \mathcal{D}\left(\mathbb{R}^{N}\right)$ with $\operatorname{supp} \psi \subseteq \bar{B}(0,4), \psi \leq 1$, and $\psi=1$ on $\bar{B}(0,2)$. We write

$$
\langle\phi, T \varphi\rangle=\langle\phi \psi, T \varphi\rangle+\sum_{j=1}^{k}\left\langle\phi\left[\psi\left(\frac{\dot{2}}{2^{j}}\right)-\psi\left(\frac{\cdot}{2^{j-1}}\right)\right], T \varphi\right\rangle+\left\langle\phi\left[1-\psi\left(\frac{\dot{2}}{2^{k}}\right)\right], T \varphi\right\rangle=A_{1}+A_{2}+A_{3}
$$

Then the weak boundedness property at 0 yields that

$$
\left\|A_{1}\right\| \lesssim \mathcal{C}_{w . b . p}(T) 2^{-k N} .
$$


Since $K$ satisfies $\left(\mathrm{SE}_{0}\right)$,

$$
\begin{aligned}
\left\|A_{3}\right\| & =\left\|\int_{\mathbb{R}^{N}} \phi(u)\left[1-\psi\left(\frac{u}{2^{k}}\right)\right] \int_{\mathbb{R}^{N}} K(u, v) \varphi(v) d v d u\right\| \\
& \leq \int_{2^{k+1}<|u|}|\phi(u)| \int_{|v| \leq 1}\|K(u, v)\||\varphi(v)| d v d u \\
& \leq \mathcal{C}_{0}(K)\|\phi\|_{L_{1}}\|\varphi\|_{L_{1}}\left(2^{k+1}-1\right)^{-N} \lesssim \mathcal{C}_{0}(K) 2^{-k N}
\end{aligned}
$$

and

$$
\begin{aligned}
\left\|A_{2}\right\| & =\left\|\sum_{j=1}^{k} \int_{\mathbb{R}^{N}} \phi(u)\left[\psi\left(\frac{u}{2^{j}}\right)-\psi\left(\frac{u}{2^{j-1}}\right)\right] \int_{\mathbb{R}^{N}} K(u, v) \varphi(v) d v d u\right\| \\
& \leq \sum_{j=1}^{k} \int_{2^{j}<|u|<2^{j+2}}|\phi(u)| \int_{|v| \leq 1}\|K(u, v)\||\varphi(v)| d v d u \\
& \leq \mathcal{C}_{0}(K) \sum_{j=1}^{k}\left(2^{j}-1\right)^{-N} \int_{2^{j}<|u|<2^{j+2}}|\phi(u)| d u\|\varphi\|_{L_{1}} \\
& \lesssim \mathcal{C}_{0}(K) k 2^{-k N} .
\end{aligned}
$$

It remains to estimate the decay of $\|\langle\phi, T \varphi\rangle\|$, if $|w|$ is large. For this we will use $\left(\mathrm{SE}_{\nu}\right)$. If $|w| \geq 2^{k+2}$, then $\phi$ and $\varphi$ have disjoint support and, since $\int_{\mathbb{R}^{N}} \phi(u) d u=0$,

$$
\langle\phi, T \varphi\rangle=\int_{\mathbb{R}^{N}} \phi(u) \int_{\mathbb{R}^{N}}[K(u, v)-K(w, v)] \varphi(v) d v d u .
$$

Hence, by $\left(\mathrm{SE}_{\nu}\right)$,

$$
\begin{aligned}
\|\langle\phi, T \varphi\rangle\| & \leq \mathcal{C}_{\nu}(K) \int_{|w-u|<2^{k}}|\phi(u)| \int_{|v|<1} \frac{|u-w|^{\nu}}{|w-v|^{N+\nu}}|\varphi(v)| d v d u \\
& \leq \mathcal{C}_{\nu}(K) \frac{2^{k \nu}}{\left(\frac{1}{2}|w|\right)^{N+\nu}}\|\phi\|_{L_{1}}\|\varphi\|_{L_{1}} \\
& \lesssim \mathcal{C}_{\nu}(K) 2^{-k N}\left(\frac{|w|}{2^{k}}\right)^{-N-\nu}
\end{aligned}
$$

Lemma 4.4 Let $k \in \mathbb{N}, w \in \mathbb{R}^{N}$, and let $\varphi, \phi \in \mathcal{D}^{n}\left(\mathbb{R}^{N}\right)$ be normalized bump functions associated with $\bar{B}(0,1)$ and $\bar{B}\left(w, 2^{k}\right)$ respectively. If $T \in \mathrm{CZO}_{n+\nu}$ satisfies the weak boundedness property at 0 and the condition $T\left(u^{\alpha}\right)=0$ for all $|\alpha| \leq n$, then

$$
\left\|\left\langle\phi, T^{\prime} \varphi\right\rangle\right\| \lesssim \mathcal{C}_{2}(T)\left(2^{k}\right)^{-N-n-\nu}\left(1+\frac{|w|}{2^{k}}\right)^{-\left(n+\nu+N / t^{\prime}\right)} .
$$

Here $\mathcal{C}_{2}(T)=\max \left\{C_{w . b . p .}(T), \mathcal{C}_{n+\nu, t}(K)\right\}$.

Proof. Let us fix a function $\psi \in \mathcal{D}\left(\mathbb{R}^{N}\right)$ which is $\leq 1$, equals 1 in $\bar{B}(0,2)$ and vanishes outside $\bar{B}(0,4)$. With this choice we write

$$
\left\langle\phi, T^{\prime} \varphi\right\rangle=\left\langle\phi \psi\left(\frac{\dot{2}}{2^{k}}\right), T^{\prime} \varphi\right\rangle+\left\langle\phi\left[1-\psi\left(\frac{\dot{2}}{2^{k}}\right)\right], T^{\prime} \varphi\right\rangle=: A_{1}+A_{2}
$$

and estimate the second term $A_{2}$ with the help of Lemma 4.1:

$$
\left\|A_{2}\right\| \leq\|\phi\|_{L_{\infty}} \int_{2^{k+1}<|v|}\left\|\left(T^{\prime} \varphi\right)(v)\right\| d v \lesssim \mathcal{C}_{n+\nu, 1}(K)\left(2^{k}\right)^{-N-n-\nu}
$$


We investigate $A_{1}$ by further splitting it in two parts:

$$
A_{1}=\left\langle\left(\phi-\sum_{|\alpha| \leq n} \frac{\phi^{(\alpha)}(0)}{\alpha !}(\cdot)^{\alpha}\right) \psi\left(\frac{\dot{\bar{k}}}{2^{k}}\right), T^{\prime} \varphi\right\rangle+\sum_{|\alpha| \leq n} \frac{\phi^{(\alpha)}(0)}{\alpha !}\left\langle(\cdot)^{\alpha} \psi\left(\frac{\dot{\overline{2}}}{2^{k}}\right), T^{\prime} \varphi\right\rangle=: A_{1,1}+A_{1,2} .
$$

Since $T\left(u^{\alpha}\right)=0$ for $|\alpha| \leq n$ and $\varphi \in \mathcal{D}^{n}\left(\mathbb{R}^{N}\right)$, we have $\left\langle(\cdot)^{\alpha}, T^{\prime} \varphi\right\rangle=0$ for all $|\alpha| \leq n$. Hence $A_{1,2}$ can be estimated

$$
\begin{aligned}
\left\|A_{1,2}\right\| & \leq \sum_{|\alpha| \leq n} \frac{\left|\phi^{(\alpha)}(0)\right|}{\alpha !}\left\|\left\langle(\cdot)^{\alpha} \psi\left(\frac{\dot{2}}{2^{k}}\right), T^{\prime} \varphi\right\rangle\right\| \\
& =\sum_{|\alpha| \leq n} \frac{\left|\phi^{(\alpha)}(0)\right|}{\alpha !}\left\|\left\langle 1-\psi\left(\frac{\cdot}{2^{k}}\right),(\cdot)^{\alpha} T^{\prime} \varphi\right\rangle\right\| \\
& \leq \sum_{|\alpha| \leq n} \frac{\left(2^{k}\right)^{-|\alpha|-N}}{\alpha !} \int_{2^{k+1}<|v|}\left\|v^{\alpha}\left(T^{\prime} \varphi\right)(v)\right\| d v \\
& \lesssim \mathcal{C}_{n+\nu, 1}(K)\left(2^{k}\right)^{-N-n-\nu} .
\end{aligned}
$$

We turn to $A_{1,1}$, which we again split in two parts:

$$
\begin{aligned}
A_{1,1}= & \sum_{j=1}^{k}\left\langle\left(\phi-\sum_{|\alpha| \leq n} \frac{\phi^{(\alpha)}(0)}{\alpha !}(\cdot)^{\alpha}\right)\left[\psi\left(\frac{\dot{2^{j}}}{2^{\prime}}-\psi\left(\frac{\cdot}{2^{j-1}}\right)\right], T^{\prime} \varphi\right\rangle\right. \\
& +\left\langle\left(\phi-\sum_{|\alpha| \leq n} \frac{\phi^{(\alpha)}(0)}{\alpha !}(\cdot)^{\alpha}\right) \psi, T^{\prime} \varphi\right\rangle \\
= & A_{1,1,1}+A_{1,1,2} .
\end{aligned}
$$

Now, by Taylor's formula,

$$
\begin{aligned}
\left\|A_{1,1,1}\right\| & \lesssim \sum_{j=1}^{k} 2^{(j+2)(n+1)} \sup _{|\alpha|=n+1}\left\|\phi^{(\alpha)}\right\|_{L_{\infty}} \int_{2^{j}<|v|<2^{j+2}}\left\|\left(T^{\prime} \varphi\right)(v)\right\| d v \\
& \lesssim \mathcal{C}_{n+\nu, 1}(K)\left(2^{k}\right)^{-N-n-1} \sum_{j=1}^{k} 2^{(j+2)(n+1)}\left[\left(2^{j}\right)^{-n-\nu}+\left(2^{j+1}\right)^{-n-\nu}\right] \\
& \lesssim \mathcal{C}_{n+\nu, 1}(K)\left(2^{k}\right)^{-N-n-1} \sum_{j=1}^{k} 2^{j(1-\nu)} \\
& \lesssim \mathcal{C}_{n+\nu, 1}(K)\left(2^{k}\right)^{-N-n-\nu}
\end{aligned}
$$

Finally, to estimate the remaining term $A_{1,1,2}$, we use the weak boundedness property. Obviously both $\varphi$ and $\left(\phi-\sum_{|\alpha| \leq n} \frac{\phi^{(\alpha)}(0)}{\alpha !}(\cdot)^{\alpha}\right) \psi$ are supported in $\bar{B}(0,4)$, and $\frac{1}{4^{N}} \varphi$ is a normalized bump function associated to this ball. Concerning the other function, we note that

$$
\partial^{\beta}\left(\phi-\sum_{|\alpha| \leq n} \frac{\phi^{(\alpha)}(0)}{\alpha !}(\cdot)^{\alpha}\right)=\phi^{(\beta)}-\sum_{\alpha \geq \beta,|\alpha| \leq n} \frac{\phi^{(\alpha)}(0)}{(\alpha-\beta) !}(\cdot)^{\alpha-\beta} .
$$

Hence, for $u \in \bar{B}(0,4)$

$$
\left|\partial^{\beta}\left(\phi-\sum_{|\alpha| \leq n} \frac{\phi^{(\alpha)}(0)}{\alpha !}(\cdot)^{\alpha}\right)(u)\right| \lesssim\left(2^{k}\right)^{-N-n-1}
$$


and, from Leibniz' rule,

$$
\left|\partial^{\delta}\left[\left(\phi-\sum_{|\alpha| \leq n} \frac{\phi^{(\alpha)}(0)}{\alpha !}(\cdot)^{\alpha}\right) \psi\right]\right| \lesssim\left(2^{k}\right)^{-N-n-1} \sum_{0 \leq \beta \leq \delta}\left(\begin{array}{c}
\delta \\
\beta
\end{array}\right)\left\|\psi^{(\delta-\beta)}\right\|_{L_{\infty}}
$$

so that $\left(\phi-\sum_{|\alpha| \leq n} \frac{\phi^{(\alpha)}(0)}{\alpha !}(\cdot)^{\alpha}\right) \psi$ is $C\left(2^{k}\right)^{-N-n-1}$ times a normalized bump function associated with $\bar{B}(0,4)$, where $C$ is some constant independent of $k$. The weak boundedness property at 0 then gives

$$
\left\|A_{1,1,2}\right\| \lesssim \mathcal{C}_{w . b . p .}(T)\left(2^{k}\right)^{-N-n-1}
$$

Putting everything together, we have shown that

$$
\left\|\left\langle\phi, T^{\prime} \varphi\right\rangle\right\| \lesssim \mathcal{C}_{2}(T)\left(2^{k}\right)^{-N-n-\nu} .
$$

To get the appropriate decay in $|w|$, we need to take into account the support properties of our bump functions more carefully. For this let $R:=\frac{1}{5}|w|>2^{k}$. Since $2^{k}+4 R<5 R=|w|$, the functions $\phi$ and $\psi(\cdot / R)$ have disjoint support, which means that $\phi \psi(\cdot / R)$ vanishes on $\mathbb{R}^{N}$. Hence

$$
\left\langle\phi, T^{\prime} \varphi\right\rangle=\left\langle\phi[1-\psi(\cdot / R)], T^{\prime} \varphi\right\rangle,
$$

and by Lemma 4.1 ,

$$
\begin{aligned}
\left\|\left\langle\phi, T^{\prime} \varphi\right\rangle\right\| & \leq\|\phi\|_{L_{t^{\prime}}}\left(\int_{2 R<|v|}\left\|\left(T^{\prime} \varphi\right)(v)\right\|^{t} d v\right)^{1 / t} \\
& \lesssim 2^{k N / t^{\prime}}\|\phi\|_{L_{\infty}} \mathcal{C}_{n+\nu, t}(K)(2 R)^{-n-\nu-N / t^{\prime}} \\
& \lesssim \mathcal{C}_{n+\nu, t}(K) 2^{k N / t^{\prime}} 2^{-k N}\left(\frac{2}{5}|w|\right)^{-\left(n+\nu+N / t^{\prime}\right)} .
\end{aligned}
$$

In the proof of Lemma 2.1 we will use the following notation. For $r>0$ and $w \in \mathbb{R}^{N}$ we define the dilation and translation operators on $\mathcal{S}\left(\mathbb{R}^{N}\right)$ by

$$
\delta_{r} \varphi=r^{-N / 2} \varphi\left(r^{-1} \cdot\right), \quad \tau_{w} \varphi=\varphi(\cdot-w) .
$$

Moreover we define the continuous linear operator $T_{w}^{r}: \mathcal{S}\left(\mathbb{R}^{N}\right) \rightarrow \mathcal{S}^{\prime}\left(\mathbb{R}^{N}, \mathcal{L}(X, Y)\right)$ by

$$
\left\langle\phi, T_{w}^{r} \varphi\right\rangle=\left\langle\phi\left(\tau_{w} \delta_{r} \phi, T\left[\tau_{w} \delta_{r} \varphi\right]\right\rangle, \quad \varphi, \phi \in \mathcal{S}\left(\mathbb{R}^{N}\right)\right.
$$

With this definition we can reformulate the weak boundedness property as follows: The operator $T$ has the weak boundedness property if and only if for all normalized bump functions $\phi, \varphi$ associated with the unit ball, the set $\left\{\left\langle\phi, T_{w}^{r} \varphi\right\rangle: w \in \mathbb{R}, r>0\right\}$ is bounded.

If $T$ is a singular integral operator with associated kernel $K$ and $\phi, \varphi$ are test functions with disjoint support, then

$$
\begin{aligned}
\left\langle\phi, T_{w}^{r} \varphi\right\rangle & =r^{-N}\left\langle\phi\left(\frac{\cdot-w}{r}\right), T\left[\varphi\left(\frac{\cdot-w}{r}\right)\right]\right\rangle \\
& =r^{-N} \int_{\mathbb{R}^{N}} \int_{\mathbb{R}^{N}} \phi\left(\frac{u-w}{r}\right) K(u, v) \varphi\left(\frac{v-w}{r}\right) d v d u \\
& =r^{N} \int_{\mathbb{R}^{n}} \int_{\mathbb{R}^{N}} \phi(u) K(r u+w, r v+w) \varphi(v) d v d u .
\end{aligned}
$$

Therefore $T_{w}^{r}$ is also a singular integral operator associated with the kernel $K_{w}^{r}$ given by

$$
K_{w}^{r}(u, v)=r^{N} K(r u+w, r v+w) .
$$

If $K$ satisfies condition $\left(\mathrm{SE}_{n}\right)$, $\left(\mathrm{SE}_{n+\nu}\right)$ or $\left(\mathrm{HE}_{n+\nu, t}\right)$, then the kernels $K_{w}^{r}$ satisfy the same condition uniformly in $w, r$. In particular, if $T \in \mathrm{CZO}_{n+\nu}$, then so is $T_{w}^{r}$. It is clear from the definitions that $T\left(u^{\alpha}\right)=T^{\prime}\left(u^{\alpha}\right)=0$ for all $\alpha \leq \beta$ implies that $T_{w}^{r}\left(u^{\beta}\right)={T^{\prime}{ }_{w}^{r}}\left(u^{\beta}\right)=0$. 
Proof of Lemma 2.1. (a) We have that

$$
\langle\phi(\cdot-v), T[\varphi(\cdot-v)]\rangle=\left\langle\tau_{v} \phi, T\left[\tau_{v} \varphi\right]\right\rangle=\left\langle\delta_{1 / a} \phi, T_{v}^{a}\left[\delta_{1 / a} \varphi\right]\right\rangle
$$

with $a^{N / 2} \delta_{1 / a} \varphi \sim \bar{B}(0,1)$ and $a^{N / 2} \delta_{1 / a} \phi \sim \bar{B}\left(\frac{w}{a}, 2^{k}\right)$. So we can apply Lemma 4.3.

(b) Choose $t \in[1, \infty)$ so large that $\frac{N}{t}<n+\nu$ and set $\delta:=n+\nu-\frac{N}{t}$. Observe that

$$
\|\langle\varphi, T \phi\rangle\|=\left\|\langle\varphi, T \phi\rangle^{\prime}\right\|=\left\|\left\langle\phi, T^{\prime} \varphi\right\rangle\right\| \text {. }
$$

Now we can proceed in the same way as in (a), using Lemma 4.4.

Remark 4.5 A careful study of the proof shows that one needs the following sightly weaker version of the weak boundedness property: For each pair of normalized bump functions associated to the unit ball, the set $\left\{\left\langle\phi, T_{w}^{2^{j}} \varphi\right\rangle: w \in \mathbb{R}^{N}, j \in \mathbb{Z}\right\}$ is bounded.

Acknowledgements This research was carried out while the author held a Margarete von Wrangell scholarship at the University of Karlsruhe. The author wants to thank L. Weis and P.C. Kunstmann for interesting and very useful discussions. She also wants to thank the Department of Mathematics at the University of South Carolina for its kind hospitality in Summer 2005, where the idea to write this paper was born.

\section{References}

[1] Herbert Amann, Operator-valued Fourier multipliers, vector-valued Besov spaces, and applications, Math. Nachr. 186 (1997), 5-56.

[2] Guy David and Jean-Lin Journé, Une caractérisation des opérateurs intégraux singuliers bornés sur $L^{2}\left(\mathbf{R}^{n}\right)$, C. R. Acad. Sci. Paris Sér. I Math. 296 (1983), no. 18, 761-764.

[3] _ , A boundedness criterion for generalized Calderón-Zygmund operators, Ann. of Math. (2) 120 (1984), no. 2, 371-397.

[4] Tadeusz Figiel, Singular integral operators: a martingale approach, Geometry of Banach spaces (Strobl, 1989), London Math. Soc. Lecture Note Ser., vol. 158, Cambridge Univ. Press, Cambridge, 1990, pp. 95110.

[5] M. Frazier, Y.-S. Han, B. Jawerth, and G. Weiss, The T1 theorem for Triebel-Lizorkin spaces, Harmonic analysis and partial differential equations (El Escorial, 1987), Lecture Notes in Math., vol. 1384, Springer, Berlin, 1989, pp. 168-181.

[6] Maria Girardi and Lutz Weis, Operator-valued Fourier multiplier theorems on Besov spaces, Math. Nachr. 251 (2003), 34-51.

[7] Y.-S. Han and Steve Hofmann, T1 theorems for Besov and Triebel-Lizorkin spaces, Trans. Amer. Math. Soc. 337 (1993), no. 2, 839-853.

[8] Tuomas Hytönen, Fourier embeddings and Mihlin-type multiplier theorems, Math. Nachr. 274/275 (2004), 74-103.

[9] Tuomas Hytönen and Cornelia Kaiser, New proof of the T(1) theorem for Triebel-Lizorkin spaces, Georgian Math. J., to appear.

[10] Tuomas Hytönen and Lutz Weis, A T(1) theorem for integral transforms with operator-valued kernel, J. reine angew. Math., to appear.

[11] _ Singular integrals on Besov spaces, Math. Nachr. 279 (2006), 581-598.

[12] Cornelia Kaiser, Calderon-Zygmund operators with operator-valued kernels on Riesz potential spaces, Submitted.

[13. ㄴ. Wavelet transforms for vector-valued homogeneous Besov spaces, In preparation.

[14] Pierre Gilles Lemarié, Continuité sur les espaces de Besov des opérateurs définis par des intégrales singulières, Ann. Inst. Fourier (Grenoble) 35 (1985), no. 4, 175-187.

[15] Rodolfo H. Torres, Boundedness results for operators with singular kernels on distribution spaces, Mem. Amer. Math. Soc. 90 (1991), no. 442, viii +172.

[16] Hans Triebel, Theory of function spaces, Monographs in Mathematics, vol. 78, Birkhäuser Verlag, Basel, 1983.

[17] Lutz Weis, Stability theorems for semi-groups via multiplier theorems, Differential equations, asymptotic analysis, and mathematical physics (Potsdam, 1996), Math. Res., vol. 100, Akademie Verlag, Berlin, 1997, pp. $407-411$.

[18] , Operator-valued Fourier multiplier theorems and maximal $L_{p}$-regularity, Math. Ann. 319 (2001), no. 4, 735-758.

[19] Abdellah Youssfi, Continuité-Besov des opérateurs définis par des intégrales singulières, Manuscripta Math. 65 (1989), no. 3, 289-310. 